Artigo Revisão

\title{
Impacto da Glicemia Pós-Prandial e Otimização da Dosagem de Insulina em Refeições Ricas em Proteína e Gordura na Diabetes Tipo 1
}

\author{
Tatiana Fernandes ${ }^{a}{ }^{*}$, Ana Faria ${ }^{a}$, Helena Loureiro ${ }^{a}$ \\ ${ }^{a}$ Instituto Politécnico de Coimbra, Escola Superior de Tecnologia da Saúde de Coimbra, Coimbra, Portugal
}

\section{INFORMAÇÃO SOBRE O ARTIGO \\ Historial do artigo: \\ Received/ Recebido: 2021-04-25 \\ Accepted/Aceite: $2021-10-26$ \\ Publicado / Published: 2022-01-24 \\ (C) Autor (es) (ou seu (s) empregador (es)) e Revista SPEDM 2021. Reutilização permitida de acordo com CC BY-NC. Nenhuma reutilização comercial. \\ (C) Author(s) (or their employer(s)) and SPEDM Journal 2021. Re-use permitted under CC BY-NC. No commercial re-use.}

\section{Palavras-chave:}

Diabetes Mellitus Tipo 1;

Gorduras na Dieta;

Insulina/administração e dosagem;

Período Pós-Prandial;

Proteínas na Dieta;

Refeições.

\section{Keywords:}

Diabetes Mellitus, Type 1;

Dietary Fats;

Dietary Proteins;

Insulin/administration \& dosage;

Meals;

Postprandial Period.

\begin{abstract}
R E S U M O
O efeito hiperglicémico pós-prandial dos hidratos de carbono tem contribuído para que seja o nutriente mais estudado em indivíduos com diabetes tipo 1. Contudo, outros macronutrientes, como a proteína e a gordura podem afetar significativamente a glicémia pós-prandial.

O método da contagem de hidratos de carbono permite flexibilidade na composição das refeições, porém atingir um adequado controlo glicémico é um desafio para indivíduos com diabetes tipo 1, especialmente quando realizam refeições com considerável conteúdo de proteína e gordura.

Este artigo de revisão tem como objetivo analisar a evidência sobre o efeito de refeições ricas em proteína e gordura na resposta glicémica pós-prandial e identificar métodos alternativos de dosagem de insulina neste tipo de refeições em indivíduos com diabetes tipo 1.

Após o consumo de refeições mistas ocorre redução do aumento da glicemia pós-prandial precoce e hiperglicemia pós-prandial tardia. Nos últimos anos têm surgido estratégias e algoritmos promissores com o objetivo de melhorar os níveis de glicemia após o consumo de refeições mistas, como a contagem de unidades de proteína e gordura, além dos hidratos de carbono, e a administração de insulina em bolus de onda dupla em terapia com bomba infusora de insulina.
\end{abstract}

\section{Impact of Postprandial Glycemia and the Optimization of Insulin Dosage in Protein and Fat-Rich Meals with Type 1 Diabetes}

\section{A B S T R A C T}

The postprandial hyperglycemic effect of carbohydrates has contributed to making it the most studied nutrient in individuals with type 1 diabetes. However, other macronutrients, such as protein and fat, can significantly affect postprandial glycemia.

The method of carbohydrate counting allows flexibility in the composition of meals; however, achieving adequate glycemic control is a challenge for individuals with type 1 diabetes, especially when they eat meals with considerable protein and fat content.

This review article aims to analyze the evidence of the effect of meals rich in protein and fat on the postprandial glycemic response, and to identify alternative methods of insulin dosing in this type of meals in individuals with type 1 diabetes.

After the consumption of mixed meals, there is a reduction in the increase in early postprandial glycemia and late postprandial hyperglycemia. In recent years, promising strategies and algorithms have emerged with the aim of improving blood glucose levels after the consumption of mixed meals, such as the count of protein and fat units, beyond the carbohydrates, and the administration of insulin in a bolus of dual-wave with an insulin infusion pump.

\footnotetext{
*Autor Correspondente / Corresponding Author. E-Mail: tatiana.fm91@gmail.com (Tatiana Fernandes) Rua 5 de outubro - S.Martinho Bispo, 3046-854 Coimbra, Portugal
} 


\section{Introdução}

A diabetes tipo 1 (DT1) resulta da destruição autoimune das células beta pancreáticas que por norma causam deficiência absoluta de insulina. ${ }^{1}$ É responsável por $5 \%$ a $10 \%$ dos casos de diabetes. ${ }^{1}$

Os indivíduos com DT1 consideram a alimentação como o maior desafio no tratamento e controlo da doença dado que não existe um padrão alimentar único. ${ }^{1}$ Segundo a American Diabetes Association (ADA), ${ }^{1}$ a terapêutica médica nutricional (termo designado pela ADA) desempenha um papel absoluto no controlo da diabetes, considerando-se que cada indivíduo deva estar ativamente envolvido na educação, autocontrolo e planeamento do tratamento, incluindo a definição de um plano alimentar individualizado desenvolvido por um Nutricionista. ${ }^{2}$ A terapêutica médica nutricional (TMN) individualizada está associada a uma diminuição da hemoglobina glicada $(\mathrm{HbA} 1 \mathrm{c})$ entre $1,0 \%$ a $1,9 \%$, considerando-a como componente eficaz no tratamento e controlo da DT1.,

A TMN em adultos com diabetes mellitus tem como principais objetivos: promover um padrão alimentar saudável baseado na ingestão variada de alimentos ricos em nutrientes e porções apropriadas, no sentido de melhorar a saúde em geral e, também, conseguir e manter um peso corporal saudável, adquirir e manter um adequado controlo glicémico, pressão arterial e perfil lipídico, e atrasar e prevenir complicações da diabetes. ${ }^{1}$

A ingestão de hidratos de carbono (HC) afeta a resposta da glicose no sangue em indivíduos com diabetes. Embora a quantidade ideal da ingestão deste macronutriente na dieta seja inconclusiva, a sua monitorização é essencial na melhoria do controlo da glicemia pós-prandial. ${ }^{1}$ Uusitupa et $a l,{ }^{3}$ recomendam que indivíduos com DT1 ingiram a mesma quantidade de $\mathrm{HC}$ da população em geral, e prefiram alimentos ricos em HC complexos de elevada qualidade nutricional, como grãos integrais, fruta, vegetais e leguminosas.

O efeito do índice glicémico e da carga glicémica dos alimentos em indivíduos com diabetes tem produzido resultados mistos. No entanto, alguns estudos demostraram que o consumo de HC com menor carga glicémica provocaram diminuição da $\mathrm{HbAlc}$ entre $0,2 \%$ a $0,5 \%$.

Os hidratos de carbono têm sido o nutriente mais estudado pelo seu efeito hiperglicémico pós-prandial., ${ }^{4,5}$ No entanto, recentes evidências mostram que outros macronutrientes, como a proteína e a gordura podem afetar significativamente a glicémia pós-prandial. ${ }^{4-7} \mathrm{O}$ estudo recente sobre o efeito de refeições mistas com elevado teor de proteína e/ou gordura suportam evidências anteriores de que a resposta da glicose difere entre os indivíduos quando consumidas refeições ricas em proteína e/ou gordura juntamente com HC. ${ }^{4}$

Segundo Kaya et $a l,{ }^{4}$ a dosagem de insulina adaptada à refeição com base na contagem de HC não justifica o aumento prolongado ( $>6$ horas) da glicemia pós-prandial, particularmente após a ingestão de alimentos hipercalóricos com elevado conteúdo de proteína e gordura. Assim, a literatura sugere que pessoas com diabetes que dominam a contagem de $\mathrm{HC}$, usufruam do ensino do impacto glicémico de proteínas e gorduras. ${ }^{1,5}$

Na prática clínica, a revisão da monitorização contínua da glicose e os dados de registos alimentares de indivíduos com DT1 destacam os efeitos glicémicos de diferentes tipos de refeições, demonstrando que estratégias de dosagem de insulina baseadas apenas na contagem de HC têm limitações. ${ }^{8,9}$

Os adolescentes tendem a consumir refeições mistas com conteúdo proteico e lipídico marcante, especialmente alimentos com elevado teor de gordura (pizzas, por exemplo). Recomendações dietéticas para reduzir a frequência do consumo deste tipo de alimentos e uma abordagem adicional de insulina para quando forem ingeridos é fundamental. ${ }^{4}$

A American Diabetes Association sugere uma abordagem, ainda que cautelosa, no aumento das doses de insulina para refeições mistas com elevado teor de proteína e/ou gordura no sentido de tratar a hiperglicemia tardia após as refeições.

Este artigo tem como objetivo analisar o efeito de refeições ricas em proteína e gordura na resposta glicémica pós-prandial e identificar estratégias práticas de dosagem de insulina neste tipo de refeições em indivíduos com DT1.

\section{Controlo Glicémico e Hidratos de Carbono}

A insulinoterapia intensiva seja em regime de múltiplas injeções de insulina (MII), ou por infusão subcutânea contínua de insulina (CSII) requer a utilização de uma dose variável de insulina e o planeamento de refeições em função da quantidade de $\mathrm{HC}$ da refeição. ${ }^{6,10} \mathrm{O}$ cálculo das doses de insulina em bolus envolve o método de contagem de hidratos de carbono (considerando o rácio insulina: HC), a correção da glicemia pré-prandial através do fator de sensibilidade à insulina e a prática de exercício físico. ${ }^{6,11}$ Este procedimento permite maior flexibilidade na composição das refeições e melhor controlo glicémico comparativamente ao método de doses fixas de insulina e refeições exatas. ${ }^{6,11,12}$ No entanto, mesmo em esquema intensivo de insulinoterapia e quantificação de $\mathrm{HC}$, atingir níveis de glicemia ótimos e a variabilidade da glicose ao longo do dia torna-se um desafio. ${ }^{6,13}$

Embora os HC sejam o macronutriente predominante, nos últimos anos estudos recentes demonstraram que a proteína e a gordura também causam impacto no perfil glicémico., ${ }^{4,13,14}$ A gordura e a proteína podem aumentar a concentração de glicose no sangue até 6 horas após as refeições, ou seja, hiperglicemia pós-prandial tardia. ${ }^{4,10,15}$

\section{Efeito da Proteína e Gordura na Glicemia Pós-prandial}

Na DT1 o impacto da proteína e da gordura na glicemia pós-prandial e a necessidade de ajustar a dose de insulina na refeição tem sido controverso. Estudos mostram que o consumo de refeições compostas por proteína e gordura juntamente com $\mathrm{HC}$ reduzem o aumento da glicemia pós-prandial precoce ( 1 a 2 horas) mas promovem a hiperglicemia pós-prandial tardia (3 a 6 horas). ${ }^{4,8,16-18}$ Para Evans et al, ${ }^{12}$ refeições compostas por HC e proteínas promovem aumento da glicemia aos 90 a 120 minutos.

Os autores do estudo mostraram que após o consumo de uma refeição composta por $40 \mathrm{~g}$ de gordura, $27 \mathrm{~g}$ de proteína e $50 \mathrm{~g}$ de $\mathrm{HC}$, foi necessário aumentar a dose de insulina em $65 \% \pm 10 \% \mathrm{e}$ entregue em bolus de onda dupla dividido em 30\% e 70\% ao longo de 2,4 horas para atingir o controlo de glicose pós-prandial adequado. Salientando de que um bolus de onda dupla é mais capaz de controlar o efeito hiperglicémico de uma refeição rica em gordura. ${ }^{17}$

Um ensaio clínico randomizado delineado em crianças e adolescentes com DT1 determinou o efeito da glicemia pós-prandial em refeições hiperproteicas e hiperlipídicas com o mesmo conteúdo de HC. Verificou que as diferenças da glicose média nas várias refeições-teste eram evidentes a partir dos 120 minutos após a refeição. A média de glicemia, entre os 180 a 300 minutos, após refeição hiperproteica/hipolipídica foi significativamente maior do que a refeição hipoproteica/hipolipídica. O mesmo se obser- 
vou após o consumo de refeição hipoproteica/hiperlipídica versus hipoproteica/hipolipídica, mas em tempos diferentes (210 a 300 minutos). Por outro lado, igualmente noutros estudos, se verificou um efeito aditivo acentuado e prolongado após o consumo de refeição hiperproteica/hiperlipídica. ${ }^{4,16}$ A média da glicemia pós-prandial era significativamente mais elevada, entre os 180 a 300 minutos, em comparação a outras refeições $(p=0,04)$. Notou ainda, redução da glicemia até aos 90 minutos após o consumo da refeição hiperlipídica, provavelmente pelo efeito da gordura no atraso do esvaziamento gástrico. ${ }^{16}$

Uma revisão concluiu que o impacto da concentração de glicose pós-prandial às 3 horas numa refeição com $40 \mathrm{~g}$ de proteína e $35 \mathrm{~g}$ de gordura é equivalente ao consumo de $20 \mathrm{~g}$ de $\mathrm{HC}$ sem insulina. ${ }^{5}$

Estudos sugerem que a proteína tem efeitos distintos se consumida com ou sem HC. . $^{5-12,19,20}$ Quando a proteína foi consumida de forma isolada em quantidades $\geq$ a $75 \mathrm{~g}$, as concentrações de glicose aumentaram após 100 minutos. Em refeições com proteína e HC, o aumento da concentração de glicose foi observada após 3 a 4 horas. ${ }^{5-7,19,20}$ Assim, refeições que contenham quantidades inferiores a $75 \mathrm{~g}$ de proteína isoladamente, não necessitam de ajuste nas doses de insulina; em refeições mistas de pelo menos $40 \mathrm{~g}$ e $30 \mathrm{~g}$ de proteína e $\mathrm{HC}$, respetivamente, considera-se necessário o aumento da dose total de insulina em $15 \%$ a $20 \%{ }^{5,6,12,19,20}$ Van der Hoogt et al, ${ }^{15}$ observaram que refeições hiperlipídicas hiperproteicas exigiam significativamente mais insulina do que refeições hipolipídicas hipoproteicas, cerca de oito vezes mais insulina de correção pós-prandial (1,2 vs 0,15 unidades (U)) e 1,3 vezes (30\%) mais insulina no total da refeição $(3,24$ vs 2,7 U). A ocorrência de hipoglicemia foi significativamente maior na refeição hipolipídica hipoproteica, reconhecendo, tal como outros estudos, que a proteína é protetora de hipoglicemias. ${ }^{15,16,20,21}$ Embora a refeição hiperlipídica hiperproteica não ter originado um pico de glicose significativamente mais elevado $(p=0,14)$, o tempo para atingir o valor de glicose máximo foi mais longo (364 vs 185 minutos) e a área sob a curva (AUC) de glicose ( $>8 \mathrm{mmol} / \mathrm{L})$ significativamente maior (198 vs 46,3 mmol/L).$^{15}$ Bell et al, ${ }^{17}$ aplicando a mesma dose de insulina para os HC de cada refeição, observaram que uma refeição rica em proteína e gordura aumentou duas vezes mais a AUC glicose em comparação a uma refeição pobre em proteína e gordura. ${ }^{17,20}$

No estudo randomizado, em adultos com DT1 usando terapia com bomba CSII em bolus de onda dupla, foi comparada a glicemia pós-prandial e a necessidade de insulina em várias quantidades e tipos de gordura na dieta. Verificou-se que o aumento da quantidade de gordura não alterou significativamente a AUC glicose mas houve uma tendência significativa na diminuição da resposta glicémica pós-prandial precoce $(p<0,001)$ e aumento da resposta pós-prandial tardia $(p=0,001)$. O aumento da quantidade de gordura não causou diferença significativa no nível do pico de glicose no sangue $(p=0,014)$ mas o tempo para atingir o pico de glicemia foi progressivamente prolongado $(p=0,010)$. A incidência de hipoglicemia foi diminuindo com o aumento da quantidade de gordura na refeição, em $47 \%, 20 \%, 7 \%$ e $0 \%$ para refeições compostas por $0,20,40$ e $60 \mathrm{~g}$ de gordura, respetivamente $(p<0,001) .{ }^{22}$ Nos tipos de gordura, monoinsaturada (abacate), polinsaturada (margarina) e saturada (manteiga) não se verificou diferença significativa na AUC glicose de 5 horas. ${ }^{22}$ Este estudo sugere que a dose total de insulina necessita de ser ajustada conforme a quantidade de gordura para minimizar o risco de hipoglicemia pós-prandial precoce e hiperglicemia pós-prandial tardia. Para otimização da resposta glicémica em refeições ricas em gor- dura é necessário aumento da dose de insulina até 20\%, doseadas em bolus de onda dupla. ${ }^{22}$

Wolpert et al, ${ }^{9}$ compararam dois períodos de 18 horas de controlo de glicose, em circuito fechado, após um jantar com elevado teor de gordura $(60 \mathrm{~g})$ e outro jantar com baixo teor de gordura ( $10 \mathrm{~g})$, ambos com idêntico teor de $\mathrm{HC}$ e proteína. O jantar hiperlipídico exigiu mais insulina do que o jantar hipolipídico $(12,6 \pm 1,9$ $\mathrm{U} v s 9,0 \pm 1,3 \mathrm{U})$ e, apesar da insulina adicional, houve hiperglicemia pós-prandial (AUC $>120 \mathrm{mg} / \mathrm{dL}=16,967 \pm 2,778$ vs 8,350 $\pm 1,907 \mathrm{mg} / \mathrm{dL} \mathrm{min})$. A média de insulina necessária para o jantar com elevado teor de gordura foi de $42 \%$, porém com diferenças interindividuais marcantes. ${ }^{9}$

Gingras et $a l,{ }^{18}$ estudaram o efeito da adição de proteína e gordura em refeições com teor fixo de $\mathrm{HC}$ no controlo da glicose pós-prandial, utilizando o sistema de administração de insulina em circuito fechado. Observaram que a administração total de insulina pós-prandial foi menor na refeição padrão (composta apenas por HC) do que a refeição com adição de proteína e gordura. Em relação aos padrões de infusão de insulina, verificaram aumento da infusão de insulina 1 hora após a refeição padrão e suspensão de insulina 2 horas e meia a 4 horas depois da refeição. Por outro lado, tanto na refeição com adição de gordura como na refeição com adição de gordura e proteína, a infusão de insulina foi ligeiramente aumentada 1 hora após a refeição e mantida durante o período pós-prandial (5 horas). Os autores indicaram que em sistemas de administração de insulina em circuito fechado, a adição de proteína e/ou gordura a uma refeição composta por HC, não influencia a AUC glicose de 5 horas. Contudo a hiperglicemia pós-prandial tardia foi evidente, sendo que a magnitude do efeito foi reduzido, possivelmente pela infusão de insulina em circuito fechado compensar a hiperglicemia na fase pós-prandial tardia. ${ }^{18}$

Estudo transversal, efetuado numa grande amostra de jovens $(\mathrm{n}=252)$ com DT1, avaliou a ingestão de macronutrientes da dieta e comparou com as guidelines existentes. Observou que a maioria dos jovens realizava uma alimentação excessiva em gordura, nomeadamente saturada e trans. Em comparação, os jovens com menor ingestão de gordura e maior ingestão de fibras obtiveram melhor controlo glicémico. ${ }^{23}$

\section{Mecanismo Fisiológico}

Os hidratos de carbono quando absorvidos aumentam rapidamente as concentrações de glicose no sangue. ${ }^{8}$

A proteína apresenta um aumento atrasado e prolongado do nível da glicemia pelo efeito da conversão de aminoácidos em glicose pela via da gliconeogénese, e influência de múltiplas hormonas, como glucagon, cortisol, hormona de crescimento, IGF1 e grelina que afetam a homeostase da glicose, aumentando a insulinorresistência. ${ }^{4,8,12-14,16,19}$ Seja em indivíduos com ou sem DT1 após uma refeição rica em proteína ocorre estimulação da secreção de glucagon. Em indivíduos saudáveis o impacto na glicemia pós-prandial é mínimo, devido à neutralização dos efeitos da libertação da insulina pela estimulação concomitante de glucagon. ${ }^{13}$ Porém, em indivíduos com DT1, a carência quase absoluta de insulina e o aumento do glucagon causam hiperglicemia pós-prandial. ${ }^{13,24}$ Alterações hormonais como o aumento do cortisol, hormona de crescimento, IGF-1 e diminuição dos níveis de grelina ocorrem após uma refeição com elevado teor de proteína. ${ }^{13}$

Tal como a proteína, a gordura também retarda a resposta glicémica. Os ácidos gordos livres levam ao aumento da resistência à insulina e hormonas como glucagon, GLP-1, polipéptido inibi- 


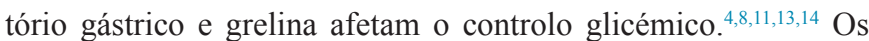
triglicéridos da gordura são metabolizados em glicerol podendo ser usado pela via da gliconeogénese. ${ }^{8,11,13}$ Os mecanismos que contribuem para a hiperglicemia gerados pela gordura dietética e ácidos gordos livres são a sensibilidade à insulina prejudicada e o aumento da produção de glicose hepática, simultaneamente com o

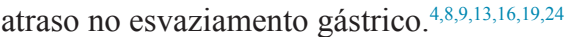

\section{Otimização da Dosagem de Insulina em Refeições Mistas}

Até ao momento, não existem diretrizes definidas para a contabilização da proteína e gordura existente nos alimentos e nas refeições como um fator importante na glicemia pós-prandial em indivíduos com DT1. Habitualmente, na terapêutica com bomba CSII e na terapêutica com MII, as doses de insulina são determinadas apenas pela quantidade total de $\mathrm{HC}$ ingeridos numa refeição e são administrados num único bolus imediato, sem ajuste no componente da proteína e gordura da refeição. ${ }^{25}$

Nos últimos anos, vários estudos recomendam o cálculo da dose de insulina necessária para uma refeição mista, baseado na contagem de unidades de proteína e gordura, além dos HC.4,26 Inicialmente a administração em bolus imediato, da insulina necessária para cobrir a quantidade de $\mathrm{HC}$ existente na refeição e, posteriormente a dose de insulina para a proteína e gordura num bolus estendido. ${ }^{4,26}$

A dose total de insulina em bolus de onda dupla, ou seja a distribuição de bolus de onda normal e bolus de onda quadrada combinados, demonstram menor valor de glicose aos 240 minutos após uma refeição rica em gordura e proteína.26 No estudo de Lopez et al, ${ }^{21}$ a utilização de um bolus combinado de 70/30 (70\% normal $/ 30 \%$ estendido) e 60/40 (60\% normal $/ 40 \%$ estendido) controlaram a glicose até aos 120 minutos. No entanto, aos 240 a 300 minutos pós-prandiais, a área de glicose sob a curva foi significativamente menor num bolus combinado de 30/70 (30\% normal $/ 70 \%$ estendido).

Até ao momento, os bolus de insulina combinados para refeições com elevado teor de proteína e gordura são recomendados para indivíduos com DT1 em terapia com bomba CSII mas em terapia com MII os resultados não são claros. ${ }^{10,24,27}$ Paterson et al, ${ }^{13}$ sugeriram comparar a insulina regular com a insulina de ação rápida em refeições ricas em gordura e/ou proteína, considerando, em teoria, que a insulina regular com início de ação mais lento e duração de ação prolongado fosse vantajosa. No entanto, Jabłońska et $a l,{ }^{10}$ concluiram que não existia benefício em cobrir este tipo de refeições com insulina regular em indivíduos com DT1 tratados com MII. Outros autores referem que na monitorização dos níveis de glicemia se ocorrer hiperglicemia tardia, deve ser administrado um bolus adicional de insulina 60 a 90 minutos após a refeição, ${ }^{17,24}$ além da combinação de insulina pré-prandial 15 a 20 minutos. ${ }^{5,24}$

Segundo Piechowiak et $a l^{14}$ e Pańkowska et $a l,{ }^{26}$ todos os participantes dos estudos que receberam insulina em bolus de onda dupla e calcularam as doses de insulina usando os algoritmos para HC, proteína e gordura, apresentaram um nível de glicose significativamente mais baixo nas 2 a 6 horas após a refeição. Verificou-se o oposto no grupo que contabilizou a dose de insulina necessária apenas para os $\mathrm{HC}$ e utilizou bolus de onda normal. ${ }^{26}$ Estes autores observaram ainda que os indivíduos que utilizaram bolus de onda dupla, apresentaram nível de glicose significativamente menores nas 4 a 12 horas após a administração de insulina. ${ }^{26}$

Van der Hoogt et al,,$^{15}$ determinaram que a necessidade total de insulina para refeições mistas (teor de gordura e proteína va- riável mas teor de $\mathrm{HC}$ constante), usando terapia com bomba de insulina e monitorização contínua da glicose em crianças com DT1, aumentou 0,12 e 0,24 unidades por cada grama de proteína e gordura, respetivamente. Logo, uma unidade adicional de insulina de correção por cada $8 \mathrm{~g}$ de proteína e uma unidade adicional de insulina de correção por cada $4 \mathrm{~g}$ de gordura, numa refeição mista que contenha HC. Esta proporção de 2:1 revela a combinação da gordura e proteína em que $1 \mathrm{~g}$ de gordura tem o dobro do efeito de $1 \mathrm{~g}$ de proteína. ${ }^{15}$ Este estudo mostra que todos os macronutrientes requerem insulina, considerando que a adição de $1 \mathrm{~g}$ de gordura requer o dobro da dose de insulina de correção comparativamente a cada grama de proteína. ${ }^{15}$

Segundo Pańkowska et $a l,{ }^{28}$ refeições compostas por proteína e gordura requerem ser "cobertas" por insulina, e a dose de insulina deve ser programada em bolus de onda normal para os $\mathrm{HC}$ e em bolus de onda quadrada para a proteína e/ou gordura. Associada a esta abordagem, os autores criaram o método unidade de gordura-proteína (fat-protein unit - FPU) para estimar as necessidades de insulina para a ingestão de gordura ou proteína. Uma FPU é definida como $100 \mathrm{Kcal}$ presentes em gordura e/ou proteína, sendo equivalente a 10 gramas de HC. Assim, a dose de insulina necessária no bolus de onda normal deve ser calculada com base no número de unidades de $\mathrm{HC}$, enquanto a dose para o bolus de onda quadrada deve ser calculada através do número de FPUs, considerando os rácios insulina: HC e insulina: FPU (dose de insulina que cobre $10 \mathrm{~g}$ de $\mathrm{HC}$ e $100 \mathrm{Kcal}$ de gordura-proteína). Atendendo à resposta pós-prandial tardia em refeições ricas em proteína e gordura, a duração do bolus de onda quadrada foi programado em 3 horas para refeições que incluía 1 FPU, 4 horas para 2 FPUs, 5 horas para 3 FPUs e 8 horas para mais de 3 FPUs. ${ }^{28}$ Associada a esta metodologia, crianças e adolescentes que usaram pelo menos uma vez por dia um bolus de onda dupla ou onda quadrada obtiveram um melhor controlo metabólico (HbA1c). ${ }^{28}$

Estudos avaliaram a eficácia da contagem de $\mathrm{HC}$ e de gordura e proteína (segundo a equação de Pankowska et $a l^{28}$ ) após refeições mistas em indivíduos com diabetes tipo 1 com CSII, e verificaram que os valores de glicose pós-prandial e AUC foram significativamente menores em comparação à contagem de HC convencional, ${ }^{4,27,29}$ independentemente do tipo de bolus. ${ }^{27}$ Isto revela que o uso de bolus normal com base na contagem de $\mathrm{HC}$ convencional não atinge níveis de glicemia pós-prandial ótimos, ${ }^{4,27}$ mesmo em terapia de insulina em circuito fechado. 30 Os episódios de hipoglicemia foram mais frequentes no método de contagem de $\mathrm{HC}$, proteína e gordura do que na contagem de $\mathrm{HC}$ convencional. ${ }^{6,11,12,27,29,31,32}$ Pańkowska et $a l^{32}$ e Kordonouri et al, ${ }^{27}$ justificam que o evento se deve à elevada proporção de insulina basal que não foi ajustada de acordo com o protocolo do estudo (Tabela 1).

\section{Conclusões e Análise Crítica}

A evidência de que refeições ricas em proteína e gordura afetam o controlo glicémico acarreta implicações importantes no aconselhamento ao doente. Nos últimos anos surgiram estratégias e algoritmos de otimização da dosagem de insulina para refeições mistas mas a sua aplicação envolve profissionais de saúde treinados e diabéticos tipo 1 capazes. Assim, a contínua investigação estabelecendo etapas exequíveis é essencial na abordagem clínica., ${ }^{9,27}$

Até ao momento, os resultados das investigações são promissores mas os autores dos estudos admitem algumas limitações, tais como a variabilidade genética, a duração da diabetes, a idade, 
Tabela 1. Recomendações clínicas de otimização da dosagem de insulina em refeições ricas em proteína e gordura na DM1.

Instituir TMN e envolver o indivíduo com DM1 na educação, autocontrolo e planeamento do tratamento. ${ }^{1}$

Capacitação para o método de contagem de HC e cálculo das doses de insulina em bolus. 1,5,6,11

Revisão da monitorização contínua da glicose e identificação de alimentos e/ou refeições com efeito hiperglicémico pós-prandial. ${ }^{8,9}$

Recomendações dietéticas para reduzir consumo de alimentos hiperglicémicos. ${ }^{4}$

Abordagem adicional de insulina para quando ingeridos alimentos ricos em proteína e/ou gordura. ${ }^{4}$

Proteína

Gordura

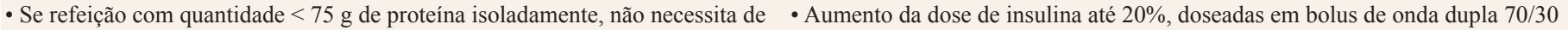
ajuste nas doses de insulina. 5,6,12,19,20 $(70 \%$ normal $/ 30 \%$ estendido $) .^{21,22}$

- Refeições mistas de pelo menos $40 \mathrm{~g}$ de proteína e $30 \mathrm{~g}$ de $\mathrm{HC}$, aumentar a

dose total de insulina em $15 \%$ a $20 \%$. $^{5,6,12,19,20}$

Adição de $1 \mathrm{U}$ de insulina de correção por cada $8 \mathrm{~g}$ de proteína e $1 \mathrm{U}$ de insulina de correção por cada $4 \mathrm{~g}$ de proteína. ${ }^{15}$

Método FPU ${ }^{28}$

$1 \mathrm{FPU}=100 \mathrm{Kcal}$ em proteína e/ou gordura

Dosagem de insulina, em bolus de onda normal, calculada com base no número de unidades de HC

e em bolus de onda quadrada calculada através do número de FPUs.

Programação do bolus de onda quadrada em:

3 horas para refeições com 1 FPU,

4 horas para refeições com 2 FPU's,

5 horas para refeições com 3 FPU's,

8 horas para refeições com mais de 3 FPU's.

Em MII, administrar um bolus adicional de insulina 60 a 90 minutos após refeição, além da combinação de insulina pré-prandial 15 a 20 minutos. ${ }^{5,24}$

TMN: terapêutica médica nutricional; DM1: diabetes tipo 1; HC: hidratos de carbono; g: gramas; U: unidade; FPU: fat-protein unit; Kcal: quilocalorias; MII: múltiplas injeções de insulina.

o índice de massa corporal, o género, a variabilidade individual nas necessidades de insulina e no impacto dos macronutrientes na glicemia pós-prandial, e os períodos de monitorização pós-prandial curtos. ${ }^{5,14,15,17,28}$

\section{Responsabilidades Éticas}

Conflitos de Interesse: Os autores declaram não possuir conflitos de interesse.

Suporte Financeiro: O presente trabalho não foi suportado por nenhum subsidio o bolsa ou bolsa.

Proveniência e Revisão por Pares: Não comissionado; revisão externa por pares.

\section{Ethical Disclosures}

Conflicts of Interest: The authors have no conflicts of interest to declare.

Financial Support: This work has not received any contribution grant or scholarship.

Provenance and Peer Review: Not commissioned; externally peer reviewed.

\section{References / Referências}

1. American Diabetes Association. Standards of Medical Care in Diabetes 2020. Diabetes Care. 2020;43. doi:10.2337/dc12-s011

2. Franz MJ, MacLeod J, Evert A, Brown C, Gradwell E, Handu D, et al. Academy of Nutrition and Dietetics Nutrition Practice Guideline for Type 1 and Type 2 Diabetes in Adults: Systematic Review of Evidence for Medical Nutrition Therapy Effectiveness and Recommendations for Integration into the Nutrition Care Process. J Acad Nutr Diet.
2017;117:1659-79. doi:10.1016/j.jand.2017.03.022

3. Uusitupa M, Schwab U. Evolving nutritional therapy for diabetes mellitus. Nutrients. 2020;12:12-5. doi:10.3390/nu12020423

4. Kaya N, Kurtoğlu S, Gökmen Özel H. Does meal-time insulin dosing based on fat-protein counting give positive results in postprandial glycaemic profile after a high protein-fat meal in adolescents with type 1 diabetes: a randomised controlled trial. J Hum Nutr Diet. 2020;33:396403. doi:10.1111/jhn.12711

5. Bell KJ, Smart CE, Steil GM, Brand-Miller JC, King B, Wolpert HA. Impact of fat, protein, and glycemic index on postprandial glucose control in type 1 diabetes: implications for intensive diabetes management in the continuous glucose monitoring era. Diabetes Care. 2015;38:1008-15. doi: $10.2337 / \mathrm{dc} 15-0100$

6. Hibbert-Jones E. Fat and protein counting in type 1 diabetes. Pract Diabetes. 2016;33:243-7. doi:10.1002/pdi.2049

7. Paterson MA, Smart CEM, Lopez PE, McElduff P, Attia J, Morbey C, et al. Influence of dietary protein on postprandial blood glucose levels in individuals with Type 1 diabetes mellitus using intensive insulin therapy. Diabet Med. 2016;33:592-8. doi:10.1111/dme.13011

8. Smart CEM, King BR, Lopez PE. Insulin dosing for fat and protein: is it time? Diabetes Care. 2020;43:13-5. doi:10.2337/dci19-0039

9. Wolpert HA, Atakov-Castillo A, Smith SA, Steil GM. Dietary fat acutely increases glucose concentrations and insulin requirements in patients with type 1 diabetes: Implications for carbohydrate-based bolus dose calculation and intensive diabetes management. Diabetes Care. 2013;36:810-6. doi:10.2337/dc12-0092

10. Jabłońska K, Molęda P, Safranow K, Majkowska L. Rapid-acting and regular insulin are equal for high fat-protein meal in individuals with type 1 diabetes treated with multiple daily injections. Diabetes Ther. 2018;9:339-348. doi:10.1007/s13300-017-0364-2

11. Maahs DM, Higgins J. Is Carbohydrate counting enough? towards perfection or unwanted complexity? Diabetes Technol Ther. 2012;14:3-5. doi:10.1089/dia.2011.0234

12. Evans M, Smart CEM, Paramalingam N, Smith GJ, Jones TW, King BR, et al. Dietary protein affects both the dose and pattern of insulin delivery required to achieve postprandial euglycaemia in Type 1 diabetes: a randomized trial. Diabet Med. 2019;36:499-504. doi:10.1111/dme.13875

13. Paterson M, Bell KJ, O'Connell SM, Smart CE, Shafat A, King B. The role of dietary protein and fat in glycaemic control in type 1 diabetes: implications for intensive diabetes management. Curr Diab Rep. 
2015;15:1-9. doi:10.1007/s11892-015-0630-5

14. Piechowiak K, Dżygało K, Szypowska A. The additional dose of insulin for high-protein mixed meal provides better glycemic control in children with type 1 diabetes on insulin pumps: randomized cross-over study. Pediatr Diabetes. 2017;18:861-8. doi:10.1111/pedi.12500

15. van der Hoogt M, van Dyk JC, Dolman RC, Pieters M. Protein and fat meal content increase insulin requirement in children with type 1 diabetes - Role of duration of diabetes. J Clin Transl Endocrinol. 2017;10:15-21. doi:10.1016/j.jcte.2017.10.002

16. Smart CEM, Evans M, O'Connell SM, McElduff P, Lopez PE, Jones TW, et al. Both dietary protein and fat increase postprandial glucose excursions in children with type 1 diabetes, and the effect is additive. Diabetes Care. 2013;36:3897-902. doi:10.2337/dc13-1195

17. Bell KJ, Toschi E, Steil GM, Wolpert HA. Optimized mealtime insulin dosing for fat and protein in type 1 diabetes: application of a model-based approach to derive insulin doses for open-loop diabetes management. Diabetes Care. 2016;39:1631-4. doi:10.2337/dc15-2855

18. Gingras V, Bonato L, Messier V, Roy-Fleming A, Smaoui MR, Ladouceur $\mathrm{M}$, et al. Impact of macronutrient content of meals on postprandial glucose control in the context of closed-loop insulin delivery: A randomized cross-over study. Diabetes, Obes Metab. 2018;20:2695-9. doi:10.1111/ dom. 13445

19. Paterson MA, Smart CE, Lopez PE, Howley P, McElduff P, Attia J, et al. Increasing the protein quantity in a meal results in dose-dependent effects on postprandial glucose levels in individuals with Type 1 diabetes mellitus. Diabet Med. 2017;34:851-4. doi:10.1111/dme.13347

20. Paterson MA, King BR, Smart CE, Smith T, Rafferty J, Lopez PE. Impact of dietary protein on postprandial glycaemic control and insulin requirements in Type 1 diabetes: a systematic review. Diabet Med. 2019;36:1585-99. doi:10.1111/dme.14119

21. Lopez PE, Smart CE, McElduff P, Foskett DC, Price DA, Paterson MA, et al. Optimizing the combination insulin bolus split for a high-fat, highprotein meal in children and adolescents using insulin pump therapy. Diabet Med. 2017;34:1380-4. doi:10.1111/dme.13392

22. Bell KJ, Fio CZ, Twigg S, Duke SA, Fulcher G, Alexander K, et al. Amount and type of dietary fat, postprandial glycemia, and insulin requirements in type 1 diabetes: a randomized within-subject trial. 2019;:1-8. doi:10.2337/ dc19-0687/-/DC1.

23. Katz ML, Mehta S, Nansel T, Quinn H, Lipsky LM, Laffel LM.
Associations of nutrient intake with glycemic control in youth with type 1 diabetes: differences by insulin regimen. Diabetes Technol Ther. 2014;16:512-8. doi:10.1089/dia.2013.0389

24. Evert AB. Factors beyond carbohydrate to consider when determining meantime insulin doses: protein, fat, timing, and technology. Diabetes Spectr. 2020;33:149-55. doi:10.2337/ds20-0004

25. Jones SM, Quarry JL, Caldwell-Mcmillan M, Mauger DT, Gabbay RA. Optimal insulin pump dosing and postprandial glycemia following a pizza meal using the continuous glucose monitoring system. Diabetes Technol Ther. 2005;7:233240. doi:10.1089/dia.2005.7.233

26. Pańkowska E, Błazik M, Groele L. Does the fat-protein meal increase postprandial glucose level in type 1 diabetes patients on insulin pump: the conclusion of a randomized study. Diabetes Technol Ther. 2012;14:16-22. doi:10.1089/dia.2011.0083

27. Kordonouri O, Hartmann R, Remus K, Bläsig S, Sadeghian E, Danne T. Benefit of supplementary fat plus protein counting as compared with conventional carbohydrate counting for insulin bolus calculation in children with pump therapy. Pediatr Diabetes. 2012;13:540-4. doi:10.1111/j.1399-5448.2012.00880.x

28. Pańkowska E, Szypowska A, Lipka M, Szpotańska M, Błazik M, Groele L. Application of novel dual wave meal bolus and its impact on glycated hemoglobin A1c level in children with type 1 diabetes. Pediatr Diabetes. 2009;10:298-303. doi:10.1111/j.1399-5448.2008.00471.x

29. Lopez PE, Evans M, King BR, Jones TW, Bell K, McElduff P, et al. A randomized comparison of three prandial insulin dosing algorithms for children and adolescents with Type 1 diabetes. Diabet Med. 2018;35:14407. doi:10.1111/dme.13703

30. Gillingham MB, Li Z, Beck RW, Calhoun P, Castle JR, Clements M, et al. Assessing mealtime macronutrient content: patient perceptions vs expert analyses via a novel phone App. Diabetes Technol Ther. 2021;23:85-94. doi:10.1089/dia.2020.0357

31. Herron A, Sullivan C, Brouillard E, Steenkamp D. Late to the party: importance of dietary fat and protein in the intensive management of type 1 diabetes. A Case Report. J Endocr Soc. 2017;1:1002-5. doi:10.1210/ js.2017-00158

32. Pańkowska E, Kordonouri O. The complex food counting system in managing children and young people with type 1 diabetes. Diabetes Care Child Young People. 2013;2:68-70. 